\title{
The tantalum screw for treating femoral head necrosis: rationale and results
}

\author{
R. Aldegheri $\cdot$ G. Taglialavoro $\cdot$ A. Berizzi
}

Received: 20 June 2007 / Accepted: 8 November 2007/Published online: 7 December 2007

(C) Springer-Verlag 2007

\begin{abstract}
Femoral head necrosis (FHN) is a progressive pathology due to the failure of blood supply to the proximal femoral epiphysis, with consequent necrosis of the subchondral bone and collapse of the articular cartilage and loss of congruity between the head and the acetabulum. Borrowing the biological and mechanical principles from the vascularized fibular graft technique for the femoral neck, the tantalum screws have been introduced. They show an extraordinary porosity, osteoconductivity, biocompatibility and very good osteoinductivity. Vitreous tantalum can be processed to take the form of a screw, with a round medial extremity and a $25 \mathrm{~mm}$ threaded lateral extremity that can be inserted into the neck of the femur, thereby supporting the articular cartilage, stimulating the repair process, interrupting the interface between necrotic and healthy tissue and favoring local vascularization. We have drawn up a treatment protocol for early-stage FHN, based on the insertion of a tantalum screw into the femoral neck. The implant has a cylindrical shape, with a $10 \mathrm{~mm}$ diameter in the smooth part and $15 \mathrm{~mm}$ in the threaded part. It is available in different sizes from 70 to $130 \mathrm{~mm}$, with $5 \mathrm{~mm}$ increments. The aim of the study is to describe the clinical and instrumental results of the tantalum screw for FHN. From June 2004 to June 2006 we performed 15 implants. The tantalum screw was inserted with an incision on the trochanteric region with traction and under X-ray control. For the clinical evaluation of the hip, we used the Harris hip score (HHS). For diagnosis and staging we used standard X-rays in two views and/or MRI, using the
\end{abstract}

R. Aldegheri · G. Taglialavoro $(\bowtie) \cdot$ A. Berizzi

Orthopaedic Clinic of the Padua University,

Via Giulio Alessio 21, 35121 Padova, Italy

e-mail: giuseppe.taglialavoro@unipd.it
Steinberg classification (J Bone Joint Surg Br 77:34-41, 1995) and CT. In all cases, the osteonecrosis extended to not more than $30 \%$ of the joint surface and the cartilage was intact with no collapse. To assess the results, we compared the pre-operative and the post-operative HHS, calculated the percentage differences between the two. We then compared X-rays, CT scans and MRI before and some time after the operation to assess whether the problem had been addressed, taking into account the intracancellous edema and the possible extension of necrosis. After an average follow-up period of $15.43 \pm 5.41$ months, ten implants (seven patients out of ten) were examined and all but one patient showed a marked improvement in HHS (the average increase was $127.9 \%$ ), with no further progression of the disease. We believe that this procedure can be suitable for young patients with limited first or second stage osteonecrosis. The objective for the foreseeable future is to resolve the pain, improve the quality of life and prevent or at least postpone arthroplasty.

Keywords Femoral head necrosis · Hip ·

Surgical treatment

\section{Introduction}

Femoral head necrosis (FHN) is a progressive pathology, either primary or secondary to systemic or localized diseases whose common feature is the failure of blood supply to the proximal femoral epiphysis, with consequent necrosis of the sub-chondral bone and collapse of the articular cartilage and loss of congruity between the head and the acetabulum.

This pathology is also known as bone coronary disease. Indeed, the anatomical areas of the femoral epiphysis most 
often involved (the upper and the anterolateral ones) are characterized by a reduced vascular supply and a forced retrograde flow towards the trochanteric region, because of the extensive articular cartilage [1].

The treatment of this pathology, characterized by pain and limited function which are closely correlated with the stage of the disease, depends on the patient's age, type of occupation, etiology, degree of articular function, onset and characteristics of pain, the possibile extension or bilateral nature of the disease and general metabolic status.

Based on these considerations and above all on the stage of the disease, there are three possible treatment options: non-invasive treatment (non weight-bearing; joint mobility with passive and active exercises, magnetic field therapy, hyperbaric therapy); conservative surgical treatment (cervico-cephalic perforations; core decompression; autoplastic grafts; arthrodiatasis; osteotomy); replacement surgery (endoprosthesis; arthroplasty) [2].

When the aim is to resolve the pain and stop the evolution of the disease and hence prevent arthritic degenerative changes and avoid prosthesis, early treatment is necessary in order to stop cartilage stripping and the collapse of the articular surface.

The gold standard of conservative treatment is represented by early diagnosis, subchondral bone decompression, removal of necrotic tissue, support of joint surface and activation of the repair processes. This objective can be achieved by means of different techniques, which can be more or less invasive and more or less complex.

Borrowing the biological and mechanical principles from the vascularized fibular graft technique for the femoral neck, the tantalum screws have been introduced.

Tantalum is a highly biocompatible metal, with very good osteoinductive properties. Thanks to a special industrial process, this metal can be made to emulate the trabecular structure of cancellous bone. After a special treatment tantalum, now turned into Vitreous Tantalum (98\% tantalum, 2\% vitreous carbon), acquires a structure characterized by an extraordinary porosity involving up to $80 \%$ of its volume, with evenly distributed $550 \mu \mathrm{m}$ interconnected pores. These characteristics make tantalum a highly osteoconductive material.

Vitreous tantalum can be processed to take the form of a screw, with a round medial extremity and a $25 \mathrm{~mm}$ threaded lateral extremity that can be inserted into the neck of the femur, thereby supporting the articular cartilage, stimulating the repair process, interrupting the interface between necrotic and healthy tissue and favoring local vascularization [6-11].

In the light of the above considerations we have drawn up a treatment protocol for early-stage FHN, based on the insertion of a tantalum screw into the femoral neck. This implant, marketed in Italy by Zimmer Fracture
Management under the name of On Rode, was first launched in the US market in March 1998 and in the European market on November 19, 2001.

The implant has a cylindrical shape, with a $10 \mathrm{~mm}$ diameter in the smooth part and $15 \mathrm{~mm}$ in the threaded part. It is available in different sizes from $70 \mathrm{~mm}$ to $130 \mathrm{~mm}$, with $5 \mathrm{~mm}$ increments.

The aim of the study is to describe the clinical and instrumental results of the tantalum screw for FHN.

\section{Materials and methods}

In order to analyze the possible utilization of the tantalum screw in the femoral hip osteonecrosis, we have performed an observational study, with prospective record of hip scores and retrospective analysis of imaging studies. From June 2004 to June 2006 we performed 15 implants: 5 monolateral (3 right, 2 left), 5 bilateral implants.

One implant was applied in a patient with post-traumatic osteonecrosis; one in a patient with ulcerous rectocolitis; five in kidney-transplanted patients; two in a patient with cerebral neoplasia, three in HIV+ patients; three in patients with idiopathic osteonecrosis (Table 1).

All ten patients, six males and four females, age range 28-69 years (mean age $42 \pm 12$ years) were symptomatic. One patient with bilateral FHN had already received an uncemented total hip prosthesis on the opposite side (Tables 2, 3).

For the clinical evaluation of the hip we used the Harris hip score (HHS), focusing on items related to quantification of pain and functional deficit. For diagnosis and staging we

Table 1 The number of the implants and aetiology, stage of necrosis and side NTF

\begin{tabular}{llll}
\hline No. of implants & Etiology & Stage & Side \\
\hline 1 & Post traumatic & 2 & $\mathrm{sx}$ \\
1 & Ulcerative colitis & 2 & $\mathrm{dx}$ \\
2 & Cerebral neoplasm & 1 & $\mathrm{dx}$ \\
& & 2 & $\mathrm{sx}$ \\
3 & hiv+, hcv+ & 2 & $\mathrm{dx}$ \\
& & 1 & $\mathrm{sx}$ \\
& & 1 & $\mathrm{sx}$ \\
& & 1 & $\mathrm{dx}$ \\
& Idiopatic & 2 & $\mathrm{dx}$ \\
& & 2 & $\mathrm{sx}$ \\
5 & & 1 & $\mathrm{dx}$ \\
& & 1 & $\mathrm{dx}$ \\
& & 2 & $\mathrm{dx}$ \\
& & 2 & $\mathrm{dx}$ \\
& & 2 & $\mathrm{sx}$ \\
\hline
\end{tabular}


Table 2 The surgery, date, age, sex, the side and aetiology of NTF
${ }^{\text {a }}$ Arthroprothesis on the right side

${ }^{\text {b }}$ Hip fracture

\begin{tabular}{lllllll}
\hline No. & Surname and name & Data of surgery & Age & Sex & Side & Etiology \\
\hline 1 & C.R. & $15 / 06 / 2004$ & 28 & $\mathrm{M}$ & $\mathrm{dx}$ & Idiopatic \\
2 & S.L. & $21 / 10 / 2004$ & 69 & $\mathrm{~F}$ & bil & Kidney trasplantation \\
3 & B.L. & $15 / 02 / 2005$ & 28 & $\mathrm{M}$ & $\mathrm{sx}$ & Post traumatic \\
4 & B.L. & $21 / 02 / 2005$ & 41 & $\mathrm{~F}$ & bil & hiv+, hcv+ \\
5 & B.G & $20 / 05 / 2005$ & 37 & $\mathrm{M}$ & bil & idiopatic \\
6 & G.C. & $07 / 07 / 2005$ & 42 & $\mathrm{M}$ & $\mathrm{sx}$ & hiv+, hcv+ \\
7 & V.M. & $18 / 10 / 2005$ & 44 & $\mathrm{M}$ & $\mathrm{dx}$ & Ulcerative colitis \\
8 & D.R. & $14 / 02 / 2006$ & 50 & $\mathrm{~F}$ & $\mathrm{dx}$ & Kidney trasplantation \\
9 & V.L. & $30 / 05 / 2006$ & 50 & $\mathrm{M}$ & bil & Kidney trasplantation \\
10 & P.D. & $16 / 05 / 2006$ & 34 & $\mathrm{~F}$ & bil & Cerebral neoplasm \\
& & Mean & 42 & & & \\
& & Standard deviation & 12 & & & \\
\hline
\end{tabular}

Table 3 Stage of the NTF with Herris Hip Score before surgery

\begin{tabular}{llll}
\hline No. & Surname and name & Stage & HHS before surgery \\
\hline 1 & C.R. & 1 & 42 \\
2 & S.L. & $1-1$ & 20 \\
3 & B.L. & 2 & 68 \\
4 & B.L. & $2-1$ & 28 \\
5 & B.G. & $2-2$ & 40 \\
6 & G.C. & 1 & 77 \\
7 & V.M. & 2 & 48 \\
8 & D.R. & 2 & 45 \\
9 & V.L. & $2-2$ & 38 \\
10 & P.D. & $1-2$ & 70 \\
\hline
\end{tabular}

used standard X-rays in two views, the MRI, using the Steinberg classification $[12,13]$, and the CT. Five implants were performed in the first-stage FHN, and ten in the second-stage FHN. In all the cases, the osteonecrosis extended to not more than $30 \%$ of the joint surface and the cartilage was intact with no collapse. The $\mathrm{Rx}$ controls were performed in the postoperative period and then after 3months. $\mathrm{Rx}$ and RMI were performed after 12 and 24 months.

Traction and X-ray control were used to insert the tantalum screw. Through a straight incision on the lateral aspect of the thigh, centered in the trochanteric region, it is possible to reach the point between the trochanter and the femoral diaphysis. Under X-ray control, a guide wire is introduced into the cephalic area of necrosis in the head. Then with incremental reamers a tunnel is created in the femoral neck, up to a diameter of $10 \mathrm{~mm}$. After measuring the length, the implant is inserted. This will have to be well introduced into the lateral cortex of the femur and properly tapped, thanks to the threaded part of the implant.

To assess results we compared the pre-operative and the post-operative HHS. We then compared X-rays and MRI before and after the operation to assess whether the problem had been addressed, taking into account the intracancellous edema and the possibile extension of necrosis.

\section{Results}

The mean surgical time was of $25 \mathrm{~min}$. No intra-operative and immediate post-operative complications were recorded.

Due to trauma, two patients sustained a sub-trochanteric fracture 9-10 days after surgery (Case 5 and Case 9). Both patients were operated on, to remove the implants and apply a cephalic plate with screws. Fracture union was finally achieved in both the patients, but necrosis is still present.

A third implant $\left(\right.$ Case $6^{\circ}$ ) was removed after 12 months due to persistent pain and progress of necrosis. After removing the implant a non-cemented HA-coated prosthesis was applied.

We have evaluated seven patients (ten implants). The average follow-up period was $15.43 \pm 5.41$ months. All the patients, but one (Case $6^{\circ}$ ), showed a marked improvement in HHS, with no further progression of the disease at the MRI analysis (Table 4).

\section{Discussion}

The operative technique is simple, free from risks and complications. The tantalum screw seems to produce encouraging results, above all in reducing pain, improving hip function and probably stopping the further progression of the pathology. This surgical procedure accomplishes two functions: by drilling and reaming up to the joint cartilage, it is possibile to decompress the femoral epiphysis and remove necrotic tissue; with the implant directed towards 
Table 4 Harris Hip Score before and after surgery

The positive or negative variations and their percentages are reported

${ }^{a}$ Arthroprothesis on the right side

Fig. 1 a Case No.1, C. R. Female 28-years-old. Right femoral head necrosis, idiopathic, stage 1 . HHS before surgery: 42 . b C. R. Female 28 years-old. Post-surgery checkup after 24 months. HHS after surgery: $96(+54)$

\begin{tabular}{lllllllll}
\hline No. & $\begin{array}{l}\text { Surname } \\
\text { and name }\end{array}$ & Side & $\begin{array}{l}\text { Date of } \\
\text { surgery }\end{array}$ & $\begin{array}{l}\text { HHS } \\
\text { before } \\
\text { surgery }\end{array}$ & $\begin{array}{l}\text { HHS } \\
\text { after } \\
\text { surgery }\end{array}$ & Variation & $\begin{array}{l}\text { Increase } \\
(\%)\end{array}$ & Follow-up \\
\hline 1 & C.R. & Monolat. & $15 / 06 / 2004$ & 42 & 96 & 54 & 128.6 & 24 \\
2 & S.L. & Bil. & $21 / 10 / 2004$ & 20 & 98 & 78 & 390.0 & 20 \\
3 & B.L. & Mon. & $15 / 02 / 2005$ & 68 & 88 & 20 & 29.4 & 16 \\
4 & B.L. & Bil. & $21 / 02 / 2005$ & 28 & 72 & 44 & 157.1 & 16 \\
5 & B.G. & Bil. & $20 / 05 / 2005$ & 40 & 100 & 60 & 150.0 & 13 \\
6 & G.C. & Monolat. & $07 / 07 / 2005$ & 77 & 31 & -46 & -59.7 & 11 \\
7 & V.M. & Monolat. & $18 / 10 / 2005$ & 48 & 96 & 48 & 100.0 & 8 \\
& & & Mean & 46.1 & 83.0 & & 127.9 & 15.43 \\
& & & Standard Deviation & 20.4 & 24.8 & & 138.9 & 5.41 \\
\hline
\end{tabular}

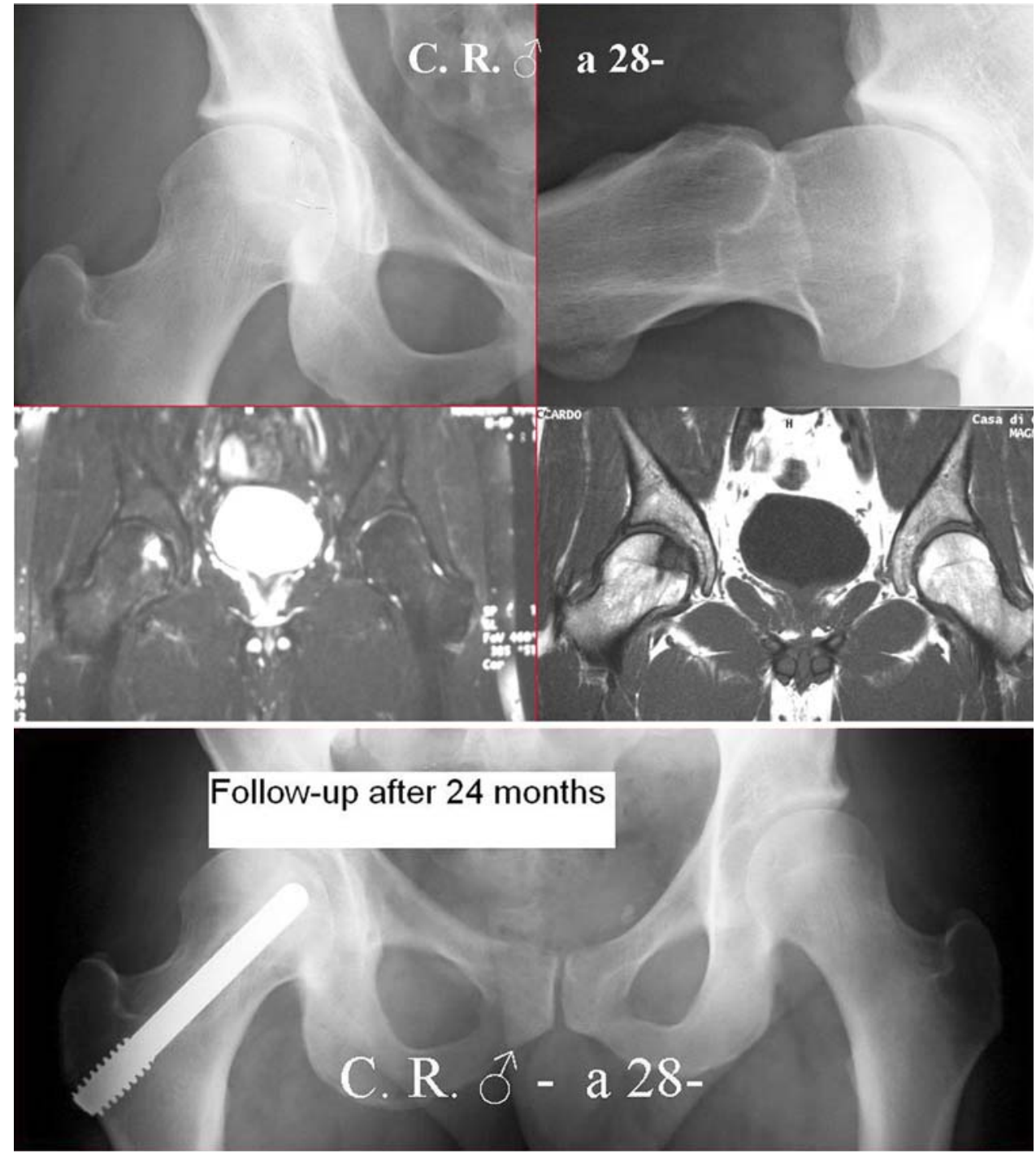

the osteonecrotic site, it is possibile to initiate the repair process and provide a mechanical support to the joint surface, as weight bearing is allowed immediately after the operation (Fig. 1). We cannot rule out that the standard diameter of $10 \mathrm{~mm}$ in the smooth part and $15 \mathrm{~mm}$ in the threaded part may somehow weaken the sub-trochanteric 
region, thereby favoring fractures at this level. In the two cases where this actually happened, the site had been subjected to trauma, but the eccentric implant in the femoral shaft may have weakened one of the two cortices at the entry site of the screw, thus favoring the fracture. This problem could be addressed with smaller diameter screws

Tantalum, in its trabecular form, combines strong mechanical resistance with high biocompatibility and osteoconductive and osteoinductive properties. For these reasons, it could be the right material for treating this pathology, which requires both a mechanical and a biological approach. We think that the tantalum screw could be indicated only in the first-and-second stage FHN, involving not more than $30 \%$ of the joint surface, indeed it shows to provide a mechanical support for the joint surface and favor post-necrotic repair processes, at the same time not ruling out a late prosthesis in case of failure (Fig. 2).
The reasons for the failure of implant Nr.6 are related to underestimation of the extent of necrosis before surgery. One single tantalum screw is unable to address not only extensive necrosis, but also first or second stage multifocal FHN, as shown. It should also be noted that the effects of the implant may be severely limited by the etiology of osteonecrosis, which may often be the consequence of a systemic pathological condition that will continue to progress even after treatment. We think that the rather high failure rate of the conservative surgical treatments could be partly due to the currently used classifications, based solely on X-rays that fail to identify FHN in its initial stages. Image diagnosis, in particular MRI and CT, can solve this problem. MRI can highlight early deterioration of the intracephalic circulation, whatever be its nature (intravasal or extravasal). CT makes it possible to accurately assess the extent of necrosis and mark the borders between the necrotic area and the healthy tissue [3-5].
Fig. 2 a Case No.6, G.C. Female 42-years-old. Biltaral femoral head necrosis. On the right side multifocal; on the left side stage 1 in $\mathrm{HIV}+/ \mathrm{HCV}+-$ patient. On the right side, due to the wide necrosis extension, we implanted a uncemented arthroprothesis; on the left side, the necrosis was stage 1 , so we implanted tantalum screw. HHS beforse surgery on the left side was 77. b G.C. Female 42years-old. Post-surgery checkup after 11 months. RX-images and MRI show an osteonecrosis evolution with collapse of the articular cartilage. HHS after surgery was $31(-46)$
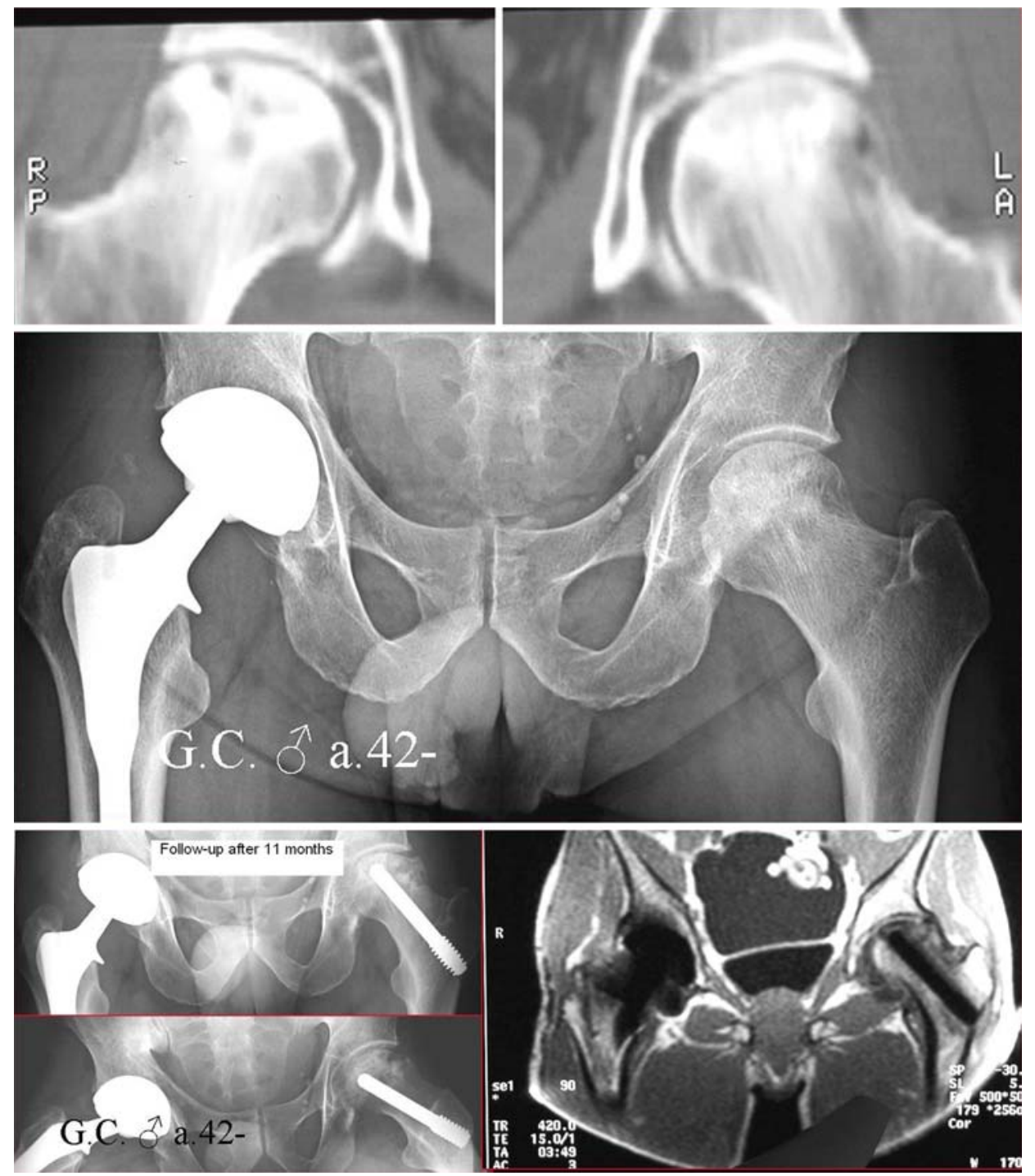
In conclusion, in the light of the above considerations we believe that this procedure can be suitable for young patients with limited first-or-second stage osteonecrosis. This treatment could be preferred to the non-invasive treatment because this one requires long periods of nonweight bearing that patients may not easily accept. Besides, only if the non-invasive treatment is started really early it can contrast the evolution of the disease (4 years after diagnosis necrosis tends to extend to $80 \%$ of the joint surface).

The objective for the foreseeable future is to resolve pain, improve the quality of life and prevent or at least postpone arthroplasty, though it may in future be possible to repair the necrotic tissue with molecular biology.

\section{References}

1. Mont MA, Hungerford DS (1995) Non-Traumatic avascular necrosis of the femoral head. J Bone Joint Surg Am 77(3):459474

2. Koo KH, Kim R, Kim YS, Ahn IO, Cho SH (2002) Risk periods of developing osteonecrosis of the femoral head in patients on steroid treatment. Clin Rheumatol 21(4):299-303

3. Sakamoto M, Shimizu K, Ida S, Akita T, Moriya H (1997) Osteonecrosis of the femoral head: a prospective study with MRI. J Bone Joint Surg Br 79(3):213-219
4. Gardeniers JM (1993) ARCO international classification of osteonecrosis. ARCO News Lett 5:79-82

5. Gardeniers JWM (1993) ARCO Commitee on Terminology and Staging. ARCO News Lett 5:79-82 Links

6. Cohen R (2002) A porous tantalum trabecular metal: basic science. Am J Orthop 31(4):216-7

7. Christie MJ (2002) Clinical applications of Trabecular Metal. Am J Orthop 31(4):219-20

8. Civinini R, Scarchini M, Villano M, Gusso MI (2003) The Rationale of a porous tantalum implant in the treatment of early stages of avascular necrosis of the femoral head-avascular necrosis of the femoral head. Current Trends pp. 85-90

9. Tsao A, Christie M, Stulberg B, Roberson JR, Hughes JH, Buckhalter RA (2003) A novel treatment for early stages of avascular necrosis, Submitted for presentation at the 2003 AAOS, New Orleans, Louisiana, USA

10. Bobyn JD, Poggie RA, Krygier JJ, Lewallen DG, Hanssen AD, Lewis RJ, Unger AS, O'Keefe TJ, Christie MJ, Nasser S, Wood JE, Stulberg SD, Tanzer M (2004) Clinical validation of a structural porous tantalum biomaterial for adult reconstruction. J Bone Joint Surg Am 86-A(Suppl 2):123-129

11. Tsao AK, Roberson JR, Christie MJ, Dore DD, Heck DA, Robertson DD, Poggie RA (2005) Biomechanical and clinical evaluations of a porous tantalum implant for the treatment of early-stage osteonecrosis. J Bone Joint Surg Am 87(Suppl 2):2227

12. Steinberg ME (1994) Early diagnosis, evaluation, and staging of osteonecrosis. Instr Course Lect 43:513-518

13. Steinberg ME, Hayken GD, Steinberg DR (1995) A quantitative system for staging avascular necrosis. J Bone Joint Surg Br 77:34-41 